\title{
Role of pharmacogenomics in the drug development
}

\section{Suvarna BS}

Assistant Professor, Department of Pharmacology, Melaka Manipal Medical College, Manipal, India

\begin{abstract}
Individuals respond differently to drugs and sometimes the effects are unpredictable. Differences in DNA that alter the expression or function of proteins targeted by drugs can contribute significantly to the variation in the individuals responses. The use of pharmacogenomics is to identify genetic polymorphisms that predispose patients to adverse drug effects that, although they may occur in only a small subset of the people treated with a new medication, are sufficiently toxic to jeopardise further development of the drug for all patients. Given the potential value of knowing all the possible factors that influence the effects of new agents, it is likely that pharmacogenomics will have an increasingly important role in drug discovery and development. This article briefly reviews concepts that underlie the emerging fields of pharmacogenetics and pharmacogenomics, with an emphasis on the pharmacogenetics of drug metabolism. Although only a few examples will be provided to illustrate concepts and to demonstrate the potential contribution of pharmacogenetics to medical practice, it is now clear that virtually every pathway of drug metabolism will eventually be found to have genetic variation.
\end{abstract}

Key words: Drug, genotype, pharmacogenetics, pharmacogenomics

$\mathrm{P}$ harmacogenetics and Pharmacogenomics play an important role in the success of drug development and therapeutics. At present pharmacogenetics studies are being done extensively for research and its application for drug development. The term pharmacogenomics comes from the words pharmacology and genomics and is thus the intersection of pharmaceuticals and genetics. The use of genetic information to explain inter-individual differences in drug responses or to individualise dosages of drugs for patients with known genetic polymorphisms is referred to as Pharmacogenomics ${ }^{1}$.

The cell components like drug metabolising enzymes, transporters and receptor, are actively involved in the drug action and any variation in genes coding can affect the drug response. An example of genetic polymorphism affecting drug metabolism would be that of the enzyme CYP2C9, which is coded by the polymorphic gene CYP2C9. Its variant alleles namely $* 2$ and $* 3$ are poor metabolisers, with only $12 \%$ and $5 \%$ respectively of enzyme activity, as compared to normal allele $* 1^{2}$.

Polymorphism of $\beta 2$ adrenergic receptor, where homozygous mutants with decreased expression of $\beta 2$ receptors do not show response to Salbutamol in asthma ${ }^{3,4}$. Polymorphisms of transporters: The promoter region of serotonin transporter gene exists in two forms: short form (sl and ss) and long form (11). The short form showed decreased clinical response to Citalopram in children or adolescents with depression or anxiety ${ }^{5}$.
Clinical observations of the patients with very high or low plasma or urinary drug concentration lead to the conception of pharmacogenetics followed by acceptance of biochemical traits suggesting that these variations are inherited. The drug metabolising enzymes were identified later which lead to the identification of the genes that encoded the proteins and the DNA sequence variation within the genes that was associated with the inherited trait ${ }^{6}$.

Most drug actions are regulated by the interaction of multiple gene products that control the pharmacokinetics and pharmacodynamics of medications, including inherited differences in drug targets and drug disposition, polygenic determinants of drug effects which becomes critical determinant in pharmacogenomics ${ }^{7}$.

\section{Drug metabolism}

There are more than 30 families of drug-metabolising enzymes in humans ${ }^{8}$ and essentially all have genetic variants, many of which translate into functional changes in the proteins encoded. To cite an example of multigenetic effect involving the CYP3A family of P-450 enzymes, in about three quarters of whites and half of

\section{Correspondence}

Dr. Beena S Suvarna

Assistant Professor, Department of Pharmacology

Melaka Manipal Medical College, Manipal, India

E-mail: drbsusheel@yahoo.co.uk 
blacks have a genetic inability to express functional CYP3A $5^{9}$. The lack of functional CYP3A5 may not be evident as many medications which are metabolized by CYP3A5 are also metabolized by the universally expressed CYP3A4. For medications that are equally metabolized by both enzymes CYP3A4 and CYP3A5; this dual pathway partially obscures the clinical effects of genetic polymorphism of CYP3A5 but contributes to the large range of total CYP3A activity in humans 9 .

\section{Drug Transporters}

Transport proteins have an important role in regulating the absorption, distribution, and excretion of many medications. Members of the adenosine triphosphate (ATP)-binding cassettefamily ofmembranetransporters ${ }^{10}$ are extensively studied transporters involved in drug disposition and effects. P-glycoprotein, a member of the ATP-binding cassette family is encoded by the human ABCB1 gene (also called MDR1). The cardinal role of P- glycoprotein is energy-dependent cellular efflux of substrates, including bilirubin, anticancer drugs, cardiac glycosides, immunosuppressive agents, glucocorticoids, human immunodeficiency virus type 1 protease inhibitors and many other medications ${ }^{11,12}$.

\section{Genetic polymorphism of drug targets}

The genetic diversity of receptors, have a profound effect on drug efficacy. Sequence variants with a direct effect on response occur in the gene for the (beta)(2))-adrenoreceptor, affecting the response to (beta)(2))-agonists ${ }^{13,14}$, arachidonate 5-lipoxygenase (ALOX5), affecting the response to ALOX5 inhibitor ${ }^{15}$; and angiotensin-converting enzyme (ACE), affecting the renoprotective actions of ACE inhibitors ${ }^{16}$. The drug-metabolizing enzymes that affect response by altering drug concentrations, such as the thiopurine methyltransferase polymorphism associated with the hematopoietic toxicity of mercaptopurine ${ }^{17}$ and susceptibility to radiation-induced brain tumours ${ }^{18}$.

Genetic variation in cellular ion transporters can also have an indirect role in predisposing patients to toxic effects of drugs. For example, patients with variant alleles for sodium or potassium transporters may have substantial morbidity or mortality resulting from druginduced long-QT syndrome ${ }^{19}$. A mutation in KCNE2, the gene for an integral membrane subunit that assembles with HERG to form $\mathrm{I}(\mathrm{Kr})$ ) potassium channels, was identified in a patient who had cardiac arrhythmia after receiving Clarithromycin ${ }^{19}$.

Genetic polymorphism in the apolipoprotein E (APOE) gene appears to have a role in predicting responses to therapy for Alzheimer's disease and to lipid-lowering drugs ${ }^{20,21}$. There are numerous allelic variants of the human APOE (APOE = apolipoprotein E) gene (e.g.,
APOE (epsilon)3, APOE (epsilon)4, APOE (epsilon)5, etc.), which contain one or more single-nucleotide polymorphisms that alter the amino acid sequence of the encoded protein. In a study of treatment of Alzheimer's disease with tacrine, 83 percent of the patients without any APOE (epsilon)4 allele showed improvement in total response and cognitive response after 30 weeks, as compared with 40 percent of patients with at least one APOE (epsilon) 4 allele ${ }^{22}$. However, the greatest individual improvement in this study was seen in a patient with a single APOE (epsilon) 4 allele, the unfavourable genotype, illustrating that a single gene will not always predict the response to a given treatment ${ }^{22}$. Follow-up studies indicate that the interaction between tacrine treatment and APOE genotype was strongest for women, again suggesting that many genes are involved in determining the efficacy of a treatment ${ }^{23}$. In another studies, patients with an APOE (epsilon) 2 allele had the greatest diminution of low-density lipoprotein cholesterol after drug therapy. The decrease was greatest with APOE (epsilon) 2, followed by APOE (epsilon) 3 and then APOE (epsilon) 4. This result was observed after treatment with multifarious lipid-lowering agents, including probucol, gemfibrozil, and many different 3-hydroxy-3-methylglutaryl-coenzymes A -reductase inhibitors(statins) ${ }^{24}$.

Genotyping of a large number of patients require methods like Amplifluor ${ }^{\circledR}$ and TaqMan ${ }^{\circledR}{ }^{6}$. When more than one genetic variation in a single chromosome is studied together methods like haplotype mapping identifies the genetic basis of diseases and newer targets for drug development ${ }^{3}$.

\section{Drug target and Pharmacogenomics}

The efficacy and safety of a drug were inadequately identified before the arrival of pharmacogenetics and proteomics. The target can be an enzyme, a receptor, a transporter, a protein in signal transduction or any protein produced in a pathological condition. After sequencing of the human genome, the number of drug targets was estimated to be around 8000, out of which 4990 could be actually acted upon - 2329 for antibodies and 794 for drug proteins ${ }^{25}$. Based on ligand binding study, 399 molecular targets belonging to 130 protein families have been identified. Currently, the number of targets ranges only around 218, though the estimated numbers are very high $^{26}$. These targets are known to exhibit variations owing to genetic polymorphisms. Variation in drug response is attributed to multiple genes rather than a single gene mutation therefore it would be appropriate to do pharmacogenomic study comparing single nucleotide polymorphism (SNP) maps and gene expression between normal and affected individuals. This identifies the genetic factors associated with the disease which could be potential future drug targets 
can be called as "tractable" or "drugable" targets 6 . Polymorphisms of $\mathrm{P} 2 \mathrm{Y}_{12}$ receptors in platelets have been identified to be associated with increased risk of coronary artery disease by haplotype analysis. This can be a potential target for a drug compound produced against coronary artery disease.

In a preliminary study, investigating the pharmacogenomics of adverse drug reactions to antifungal agents, low-density lipoproteins and cholesteryl ester transfer protein in Amphotericin B renal toxicity; toll-like receptor 1 and 2 in Amphotericin B infusion-related ADRS; phosphodiesterase 6 in voriconazole visual adverse events; flavin-containing monooxygenase, glutathione transferases and multidrug resistance proteins 1 and 2 in ketoconazole and terbinafine hepatotoxicity; CYP enzymes and P-glycoprotein in drug interactions between azoles and co administered medications; multidrug resistance proteins 8 and 9 on 5 -flucytosine bone marrow toxicity; and mast cell activation in caspofungin histamine release will help to focus on high-priority candidate genes, and understand the toxic effects of antifungal drugs identifying the candidate gene ${ }^{27}$. Tamoxifen is of clinical importance in pharmacogenomics. Tamoxifen undergoes hydroxylation to active metabolites-4hydroxytamoxifen and endoxifen (N-desmethyl-4hydroxytamoxifen) catalyzed by cytochrome P450 2D6 (CYP2D6). However, CYP2D6 is genetically polymorphic. Approximately 5-8\% of Caucasian subjects are CYP2D6 "poor metabolisers", therefore are unable to catalyze Tamoxifen hydroxylation with poorer drug response to Tamoxifen compared to CYP2D6 "extensive metabolisers ${ }^{28}$. There have also been preliminary attempts to study the pharmacogenomics of aromatase inhibitors in order to explore the nature and extent of common DNA sequence variation in the CYP19 gene that encodes aromatase ${ }^{29}$.

There is an association of the Abacavir hypersensitivity reaction with HLA- B*5701 testing patients for the allele and if present avoiding the drug can prevent the reaction. Persons with the allele CYP2B6*6 present higher Efavirenz "area under the curve" and have increased risk of neuropsychological toxicity. The genome approaches will improve the knowledge of HIV therapy in the future ${ }^{30}$. In asthma, TNF-alpha a proinflammatory cytokine has a predominant role in airway inflammation affecting the treatment outcome. The study illustrated variations of TNF-alpha gene that potentially influence asthma phenotype and highlighted some TNF- alpha blocking drugs. A cytokine specific target therapy seems to be favourable since agents that block TNF-alpha slow disease progression, suppress inflammation and in some cases induce remission of chronic inflammation ${ }^{31}$.

\section{Prediction of efficacy and safety of the drug}

The efficacy of a drug, to a great extent, is determined by appropriate target selection, which can be guided by pharmacogenomic methods. For example, the drug Trastuzumab, an anti-HER2 monoclonal antibody against metastatic breast cancer, was found to be effective only in women who were over expressing the HER2 protein ${ }^{32}$. Another example would be association between polymorphisms in the apolipoprotein $\mathrm{E}$ (APOE), cholesteryl ester transfer protein CETP, stromelysin-1 and $\beta$-fibrinogen with progression of atherosclerosis, cardiovascular events and death. It was shown that people with such polymorphisms showed good efficacy with HMG-CoA inhibitors, as compared to those without the polymorphisms ${ }^{33}$. Drug toxicity due to genetic polymorphism results in poor metabolism in an individual and therefore there are studies conducted on CYP2C9, CYP2C19 and CYP2D6 etc. as they are dependent on one or more genes ${ }^{6}$. There have been reports of emphasizing the action of Warfarin in patients with increase in INR (International Normalized Ratio) at normal doses and on pharmacogenetic testing, patients were found to be poor metabolisers with $* 3 / * 4$ and $* 1 / * 3$ polymorphisms of CYP2C9. Because of this significant association between CYP2C9 polymorphisms and Warfarin toxicity, the Food and Drug Administration (FDA) has approved inclusion of pharmacogenetic data in the product label for warfarin which states the genetic information ${ }^{34}$ and makes Warfarin a safe drug.

Expected benefits of pharmacogenomics ${ }^{35}$ :

1. Production of target specific drugs

2. Better and safe drugs

3. Drug dosage based on person's genetics

4. Vaccines made of genetic materials (DNA/ RNA) which are known to activate the immune system but not cause infection.

Shortcomings of pharmacogenomics ${ }^{35}$ :

1) Complexity of finding gene variations that affects drug response

2) Limited drug for a particular condition

3) Pharmacoeconomics

4) Educating healthcare providers (prescribing physicians) regardless of specialty will need a better understanding of genetics.

Certain guidelines have been set in the drug development for the Pharmaceutical Company which involves the submission of the pharmacogenetic data. This helps gathering scientific data by discussing the important aspects of the drug development and avoiding any delays $^{6}$. 


\section{Conclusion}

Different patients show response to an administered drug differently. Actually, drug is tested before declared fit for human use on small number of healthy volunteers and patients. But drug is consumed by large population belonging to different races and ethnic groups. Despite having authentic scientific data on drugs, one cannot be sure how the patient will respond to drug administration. The advent of Pharmacogenomics would help the physicians to prescribe the medications based on the patient's genotype thereby enhancing the efficacy and minimizing the side effects. It is unlikely that a single polymorphism in a single gene would explain a high degree of drug response variability in a consistent fashion. The need for analysis of multiple genes has been recognized and haplotype analysis and genome wide scan methods were designed.

\section{References}

1. Curtis KD. Principles of Toxicology and Treatment of Poisoning. In: Brunton L L, Lazo J S, Parker K L, editors. Goodman \& Gilman's The Pharmacological Basis of Therapeutics; 11th Ed. United States: McGraw-Hill Companies, Inc; 2006. p 1739-1751.

2. Rettie AE, et al. Impaired (S)-warfarin metabolism catalysed by the R144C allelic variant of CYP2C9. Pharmacogenetics. 1994; 4:39-42.

3. Tsai CT, Fallin D, Chiang FT, Hwang JJ, Lai LP, Hsu KL, et al. Angiotensinogen gene haplotype and hypertension: interaction with ACE gene I allele. Hypertension. 2003; 41:9-15.

4. Turki J, Pak J, Green SA, Martin RJ, Liggett SB. Genetic polymorphisms of the beta 2-adrenergic receptor in nocturnal and nonnocturnal asthma: Evidence that Gly16 correlates with the nocturnal phenotype. J Clin Invest. 1995; 95:1635-4.

5. Surendiran A, Pradhan SC, Adithan C. Role of pharmacogenomics in drug discovery and development. Indian Journal of Pharmacology. 2008; 40:137-43.

6. Evans WE et al. Pharmacogenomics - Drug Disposition, Drug Targets, and Side Effects. N Engl J of Med. 2003; 348 (6):538-49.

7. Weinshilboum R. Inheritance and drug response. N Engl J Med. 2003; 348:529-37.

8. Evans WE, Relling MV. Pharmacogenomics: translating functional genomics into rational therapeutics. Science. 1999; 286:487-91.

9. Kuehl P, Zhang J, Lin Y, et al. Sequence diversity in CYP3A promoters and characterization of the genetic basis for polymorphic CYP3A5 expression. Nat Genet. 2001; 27:383-91.
10. Borst P, Evers R, Kool M, Wijnholds J. A family of drug transporters: the multidrug resistanceassociated proteins. J Natl Cancer Inst. 2000; 92: 1295-302.

11. Choo EF, Leake B, Wandel C, et al. Pharmacological inhibition of P-glycoprotein transport enhances the distribution of HIV-1 protease inhibitors into brain and testes. Drug Metab Dispos. 2000; 28:655-60.

12. Brinkmann U, Roots I, Eichelbaum M. Pharmacogenetics of the human drug-transporter gene MDR1: impact of polymorphisms on pharmacotherapy. Drug Discov Today. 2001; 6:835-9.

13. Liggett SB. Beta (2)-adrenergic receptor pharmacogenetics. Am J Respir Crit Care Med. 2000; 161:S197-S201.

14. Dishy V, Sofowora GG, Xie H-G, et al. The effect of common polymorphisms of the (beta) $\left({ }_{2}\right)$ )-adrenergic receptor on agonist-mediated vascular desensitization. N Engl J Med. 2001; 345:1030-5.

15. Drazen JM, Yandava CN, Dube L, et al. Pharmacogenetic association between ALOX5 promoter genotype and the response to antiasthma treatment. Nat Genet. 1999; 22:168-70.

16. Jacobsen $\mathrm{P}$, Rossing $\mathrm{K}$, Rossing $\mathrm{P}$, et al. Angiotensin converting enzyme gene polymorphism and ACE inhibition in diabetic nephropathy. Kidney Int. 1998; 53:1002-6.

17. Black AJ, McLeod HL, Capell HA, et al. Thiopurine methyltransferase genotype predicts therapy-limiting severe toxicity from azathioprine. Ann Intern Med. 1998; 129:716-8.

18. Relling MV, Rubnitz JE, Rivera GK, et al. High incidence of secondary brain tumours after radiotherapy and antimetabolites. Lancet. 1999; 354:34-9.

19. Abbott GW, Sesti F, Splawski I, et al. MiRP1 forms IKr potassium channels with HERG and is associated with cardiac arrhythmia. Cell. 1999; 97:175-87.

20. Gerdes LU, Gerdes C, Kervinen K, et al. The apolipoprotein epsilon4 allele determines prognosis and the effect on prognosis of simvastatin in survivors of myocardial infarction: a sub-study of the Scandinavian Simvastatin Survival Study. Circulation. 2000; 101:1366-71.

21. Ordovas JM, Lopez-Miranda J, Perez-Jimenez F, et al. Effect of apolipoprotein E and A-IV phenotypes on the low density lipoprotein response to $\mathrm{HMG} \mathrm{CoA}$ reductase inhibitor therapy. Atherosclerosis. 1995; 113:157-66. 
22. Poirier J, Delisle MC, Quirion R, et al. Apolipoprotein E4 allele as a predictor of cholinergic deficits and treatment outcome in Alzheimer disease. Proc Natl Acad Sci U S A. 1995; 92:12260-4.

23. Farlow MR, Lahiri DK, Poirier J, Davignon J, Schneider L, Hui SL. Treatment outcome of tacrine therapy depends on apolipoprotein genotype and gender of the subjects with Alzheimer's disease. Neurology. 1998; 50:66977.

24. Siest G, Bertrand P, Herbeth B, et al. Apolipoprotein E polymorphisms and concentration in chronic diseases and drug responses. Clin Chem Lab Med. 2000; 38:84152.

25. Burgess J, Golden J. Cracking the druggable genome [Home page on the Internet]. Needham, MA USA: Bio-IT World; [updated 2002 Oct 09; cited 2008 Oct 22]. Available from http://www. bio-itworld.com/archive/100902/firstbase.html

26. Imming $P$, Sinning $C$, Meyer A. Drugs, their targets and the nature and number of drug targets. Nat Rev Drug Discov. 2006; 5:821-34.

27. Meletiadis J, Chanock S, Walsh TJ. Defining targets for investigating the pharmacogenomics of adverse drug reactions to antifungal agents. Pharmacogenomics.2008; 9(5):561-84.

28. Weinshiboum R. Pharmacogenomics of endocrine therapy in breast cancer. Adv Exp Med Biol. 2008; 630:220-31.
29. Ingle JN. Pharmacogenomics of tamoxifen and aromatase inhibitors. Cancer. 2008; $112(3$ Suppl):695-9.

30. Roca B. Pharmacogenomics of antiretrovirals. Recent Patents on Anti-Infective Drug Discovery.2008; 3(2):132-5.

31. Lykouras D et al. Role and pharmacogenomics of TNF-alpha in asthma. Mini-Reviews in Medicinal Chemistry. 2008; 8(9):934-42.

32. Shak S. Overview of the trastuzumab (Herceptin) anti-HER2 monoclonal antibody clinical program in HER2-overexpressing metastatic breast cancer: Herceptin Multinational Investigator Study Group. Semin Oncol. 1999; 26:71-7.

33. Kuivenhoven JA, et al. The role of a common variant of the cholesteryl ester transfer protein gene in the progression of coronary atherosclerosis: The Regression Growth Evaluation Statin Study Group. N Engl J Med. 1998; 338:86-93.

34. Gage BF, Lesko LJ. Pharmacogenetics of warfarin: Regulatory, scientific, and clinical issues. J Thromb Thrombolysis. 2008;25:4551.

35. American Medical Association. Pharmacogenomics [Homepage on the Internet]. Chicago: American Medical Association; c1995-2008 [updated 2008 Oct 6, cited 2008 Oct 24]. Available from http://www.ama-assn. org/ama/pub/category/2306.html 